\title{
PLUTO'S LYAPUNOV NUMBERS IN DIFFERENT DYNAMICAL MODELS
}

\author{
R. DVORAK AND E. LOHINGER \\ Institut für Astronomie, Universität Wien, \\ Türkenschanzstraße 17, A-1180 Vienna, Austria.
}

\begin{abstract}
We present the results of numerical integrations of Pluto and some fictitious Plutos in three different models (the circular and the elliptic restricted three body problem and the outer solar system). We determined the "extension" of the stable region in these models by means of the Lyapunov Characteristic Numbers and by an analysis of the orbital elements.
\end{abstract}

\section{Introduction}

Since the study of Sussman \& Wisdom $(1988,1992)$, Pluto's orbit is known to be chaotic in the sense that its Lyapunov exponent is positive. But, until now, no investigation was carried out which could show that this indication of a positive Lyapunov exponent is connected with an unstable motion. Even integrations up to half of the life-time of our solar system do not at all show significant changes in the orbital elements of Pluto (cf. Dvorak et al., 1995). The aim of this investigation is to study Pluto's motion in simple models which will be compared with more realistic ones, moreover we computed the largeness of the region in which Pluto can move without showing the above mentioned changes of the orbital elements.

\section{Three dynamical models and three different methods}

We studied Pluto's motion in the three dimensional circular restricted three body problem (Sun + Neptune), in the elliptic restricted problem and finally in a model of the outer solar system (=OSS) where the perturbations of the planets Jupiter to Neptune were taken into account. In all cases, the masses of the neglected planets were added to that of the Sun. The orbits of Pluto and 11 fictitious bodies - with semimajor axes $a=a_{P l u t o}-0.1 \cdot k$ $\mathrm{AU}, k=1, \ldots, 8 \mathrm{AU}$ and $a=a_{P l u t o}+0.1 \cdot l \mathrm{AU}, l=1,2,3$; - were ex- 

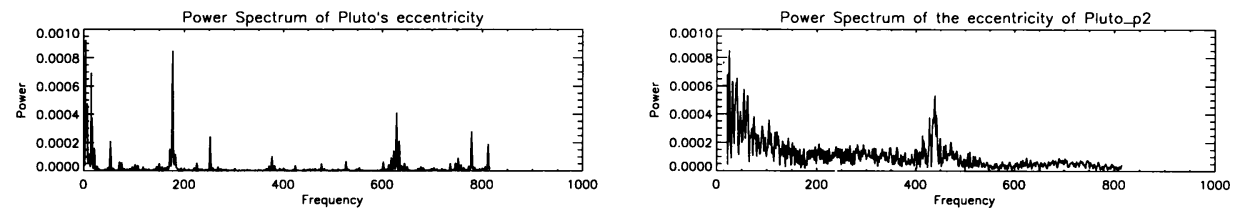

Figure 1. Fourier spectrum of the eccentricity of (a) Pluto in the model of the outer solar system and (b) of a fictitious Pluto outside the "stable" region in the restricted problem.

amined using three different approaches: $1^{\text {st }}$ by means of a FFT applied to the orbital elements, $2^{\text {nd }}$ by the calculation of the Lyapunov Characteristic Numbers $(=\mathrm{LCNs})$ and $3^{\text {rd }}$ we computed the distribution of the largest Local Lyapunov Number (LLCN) of Pluto's orbit. We have to point out that many of the studied orbits are not in 2:3 resonance with Neptune. The numerical calculations were carried out by means of two methods: the Lie integrator and the Bulirsch-Stoer integrator. The latter was used for the computation of the Lyapunov numbers - these are the local variations of the tangent vectors to the flow (Benettin et al., 1980; C. Froeschlé, 1984). In order to replace the evolved tangent vectors by new orthonormalized ones, the Gram-Schmidt procedure was employed. This program was provided by R. Gonczi (of the OCA Nice).

\section{The results}

The application of the FFT to the time evolution of the orbital elements showed that in all models Pluto's orbit has a signal which corresponds to a more or less quasiperiodic orbit over the time interval of 40 million years (see e.g. fig. 1.a). A former study of Pluto's orbital elements showed that the width of the "stability zone" of Pluto - stable in the sense that the orbital changes are not significant - turned out to be approximately $0.7 \mathrm{AU}$ in semimajor axis (cf. Dvorak et al., 1995). The present study of the Fourier spectra of the different fictitious Plutos confirmed these former results. In fig. (1.b) the signal of a chaotic orbit in the vicinity of Pluto is shown by way of comparison. The calculations of the LCNs in the two models of the restricted problem led to similar results, that is, in case of the inner bodies, the largest $\mathrm{LCN}\left(\mathrm{LCN}_{1}\right)$ is positive only for pluto_m8 (with a semimajor 

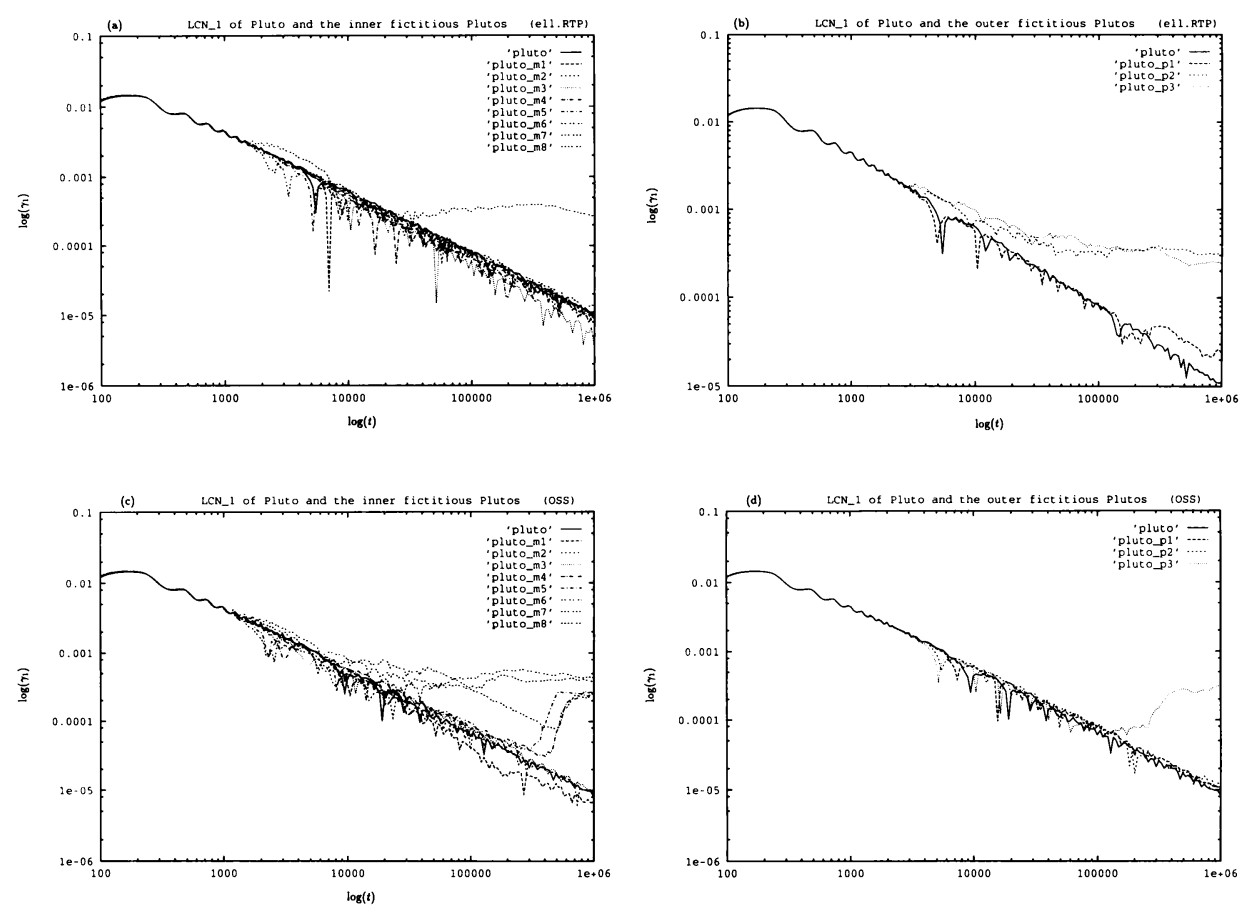

Figure 2. The largest LCNs of (a) Pluto and the "inner" Plutos, (b) Pluto and the "outer" Plutos in the spatial elliptic restricted problem and the same in the OSS (c and d). The integration time was $10^{6}$ years into the future.

axis $a=a_{\text {Pluto }}-0.8 \mathrm{AU}$ ) (see fig. 2.a) - which was even thrown out of the system. For the region outside (see fig.2.b) - outside in the sense that the initial semimajor axis is larger than Pluto's - only $\mathrm{LCN}_{1}$ of the orbit next to Pluto tends to zero within the considered time; this is consequently a quasi-periodic orbit. From the body pluto-p2 on (with a semimajor axis $a=a_{\text {Pluto }}+0.2 \mathrm{AU}$ ) the largest LCNs indicate chaotic motion. In the more realistic model (OSS) the region of stable motion seems to be shifted outwards, and became also narrower: From pluto_m4 (with a semimajor axis $a=a_{\text {Pluto }}-0.4 \mathrm{AU}$ ) on the $\mathrm{LCN}_{1}$ of all inner orbits turned out to be positive. Outside Pluto's orbit, only pluto-p3 (with a semimajor axis $a=a_{\text {Pluto }}+0.3 \mathrm{AU}$ ) has a positive $\mathrm{LCN}_{1}$. The extensions of the stable region in the restricted three body problem and in the outer solar system are presented in fig. 3 where the diminution and the shift outwards in case of the OSS is clearly seen. How does this fit to the result that Pluto's Lyapunov exponent is positive as mentioned in the introduction? We used relatively short time intervals for the determination of the LCNs: therefore 


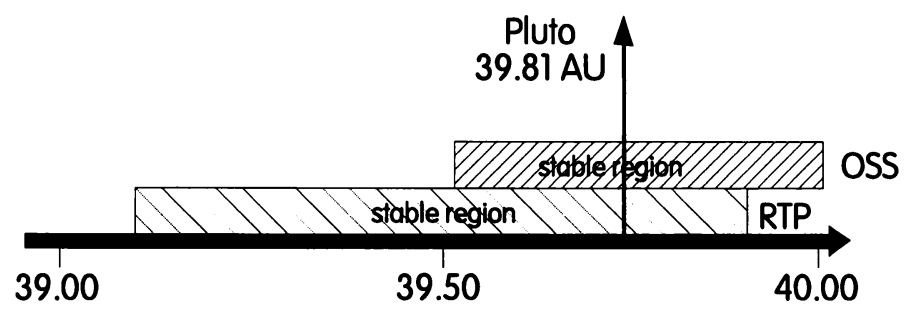

semimajor axis in $\mathrm{AU}$

ZZ outer solar system (OSS)

restricted three body problem (RTPI

Figure 3. The extension of the stable region in the restricted three body problem and in the model of the outer solar system. In the OSS this region is smaller and shifted outwards.

it was impossible to obtain a Lyapunov Number which corresponds to the Lyapunov time of about 20 million years. And still the LCNs indicate a property which is connected with our common definition of stability: our experiments show that in fact unstable motion is connected with a positive LCN. Finally we began to study the distribution of the Local Lyapunov Characteristic Numbers of the orbits in the elliptic RTP and in the OSS. For Pluto's orbit we found similar distributions for both models.A detailed analysis of the LLCNs is still in process.

\section{Acknowledgements}

The research described in this paper was supported by the Austrian Fonds zur Förderung der wissenschaftlichen Forschung (project P 9921 - AST). For travel support one of us (EL) received an IAU Travel Grant and has to thank also the HCM contract ERB4050 PL930312 which corresponds to the $F W F$ project $\mathrm{P} 10212$ - AST.

\section{References}

Benettin, G., Galgani, L., Giorgilli, A., Strelcyn, J. M. (1980) "Lyapunov Characteristic Exponents for smooth dynamical systems; a method for computing all of them", Meccanica, 15, Part I+II, 9-30.

Dvorak, R.:, Lohinger, E., Maindl, T. I. (1995) "On the chaoticity of Pluto's orbit", In Perturbation theory and chaos in nonlinear dynamics with emphasis to celestial mechanics (Hagel et al., eds.), Funchal and Vienna, pp. 20-29.

Froeschlé, C. (1984) "The Lyapunov characteristic exponents and applications", Journal de Mécanique théorique et appliquée. Numéro spécial, pp. 101-132.

Sussman, G. J., Wisdom, J. (1988) "Numerical Evidence That the Motion of Pluto Is Chaotic", Science, 241, 433-437.

Sussman, G. J., Wisdom, J. (1992) "Chaotic Evolution of the Solar System", Science, 257, 56-62. 